\title{
Existence results for a nonlocal problem involving the $\mathbf{p}(\mathbf{x})$-Laplacian
}

\author{
Mustafa Avci \\ Department of Mathematics, Dicle University, Diyarbakir, Turkey
}

Email address:

mavci@dicle.edu.tr (M. Avci)

\section{To cite this article:}

Mustafa Avci. Existence Results for A Nonlocal Problem Involving the p(x)-Laplacian. Pure and Applied Mathematics Journal. Vol. 2 , No. 1, 2013, pp. 20-27. doi: 10.11648/j.pamj.20130201.13

\begin{abstract}
In the present paper, we deal with two different existence results of solutions for a nonlocal elliptic Dirichlet boundary value problem involving $\mathrm{p}(\mathrm{x})$-Laplacian. The first one is based on the Brouwer fixed point theorem and the Galerkin method which gives a priori estimate of a nontrivial weak soltion. The second one is based on the variational methods. By using Mountain-Pass theorem, we obtain at least one nontrivial weak soltion.
\end{abstract}

Keywords: p(x)-Laplacian; Nonlocal Problem; Fixed Point Theorem; Galerkin Method; Variational Methods Mountain-Pass Theorem

\section{Introduction}

We are concerned with the following problem

$$
\begin{gathered}
-m\left(\int_{\Omega} \frac{|\nabla u|^{p(x)}}{p(x)} d x\right) \operatorname{div}\left(|\nabla u|^{p(x)-2} \nabla u\right) \\
=f(x, u) \text { in } \Omega, \\
u=0 \text { on } \partial \Omega
\end{gathered}
$$

Where $\Omega \subset \mathbb{R}^{N}$ is a smooth bounded domain, $p \in C(\bar{\Omega})$ with $1<p(x)<N$ for any $x \in \bar{\Omega}, m$ is a continuous function and $f$ is a Carathéodory function.

Problem $(\mathrm{P})$ is related to the stationary version of a model, the so-called Kirchhoff equation, introduced by [13]. To be more precise, Kirchhoff established a model given by the equation

$$
\rho \frac{\partial^{2} u}{\partial t^{2}}-\left(\frac{P_{0}}{h}+\frac{E}{2 L} \int_{0}^{L}\left|\frac{\partial u}{\partial x}\right|^{2} d x\right) \frac{\partial^{2} u}{\partial x^{2}}=0,
$$

where $\rho, h, P_{0}, E, L$ are constants which extends the classical D'Alambert's wave equation, by considering the effects of the changes in the length of the strings during the vibrations. There are papers $[4,11,15]$ in which the authors give the existence of solutions of Kirchhoff-type and p-Kirchhoff-type equations. Moreover, for $\mathrm{p}(\mathrm{x})$-Kirchhoff-type equations see, for example, [2,5-7].

The $\mathrm{p}(\mathrm{x})$-Laplace operator

$$
\operatorname{div}\left(|\nabla u|^{p(x)-2} \nabla u\right)
$$

is a natural generalization of the p-Laplacian operator

$$
\Delta_{p} u=\operatorname{div}\left(|\nabla u|^{p-2} \nabla u\right)
$$

Where

$$
p>1
$$

is a real constant. The main difference between them is that $p$-Laplacian operator is ( $p-1)$-homogenous, but the $\mathrm{p}(\mathrm{x})$-Laplacian operator, when $\mathrm{p}(\mathrm{x})$ is not constant, is not homogeneous. This causes many problems, some classical theories and methods, such as the Lagrange multiplier theorem and the theory of Sobolev spaces, are not applicable. For $\mathrm{p}(\mathrm{x})$ - Laplacian operator, we refer the readers to $[9,10,17,18]$ and references there in. Moreover, the nonlinear problems involving the $\mathrm{p}(\mathrm{x})$-Laplacian operator are extremely attractive because they can be used to model dynamical phenomenons which arise from the study of electrorheological fluids or elastic mechanics. Problems with variable exponent growth conditions also appear in the modelling of stationary thermo-rheological viscous flows of non-Newtonian fluids and in the mathematical description of the processes filtration of an ideal barotropic gas through a porous medium. The detailed application backgrounds of the $\mathrm{p}(\mathrm{x})$-Laplacian can be found in $[1,3,19,22]$ and the references there in. 
Recently, some interesting results were obtained by many authors. In [21], the authors studied a similar problem to $(\mathrm{P})$ in the case of

$$
p(x)=2
$$

They established two different existence results of solutions for a nonlocal elliptic equations with nonlinear boundary condition by using the Galerkin method and the Mountain-Pass theorem. In [16], the authors dealt with problem $(\mathrm{P})$ in the case of

$$
p(x)=2
$$

By using the Brouwer fixed point theorem and the Galerkin method, they proved the existence of a solution.

Motivated by the above references, we deal with the existence of solutions for a nonlocal elliptic equation $(\mathrm{P})$ with Dirichlet boundary condition involving the $\mathrm{p}(\mathrm{x})$-Laplacian operator. The first result is obtained by the Brouwer fixed point theorem and the Galerkin method, whereas the others are obtained in view of variational approach using the Mountain-Pass theorem.

\section{Preliminaries}

First, we recall some basic properties of spaces

$$
L^{p(x)}(\Omega)
$$

And

$$
W^{1, p(x)}(\Omega)
$$

(for details, see e.g., $[8,14])$.

Set

$$
C_{+}(\bar{\Omega})=\{p: p \in C(\bar{\Omega}), p(x)>1 \text { for any } x \in \bar{\Omega}\} .
$$

For any

$$
p \in C_{+}(\bar{\Omega})
$$

denote

$$
p^{-}:=\inf _{x \in \bar{\Omega}} p(x), p^{+}:=\sup _{x \in \bar{\Omega}} p(x)<\infty,
$$

and define the variable exponent Lebesgue space by

$L^{p(x)}(\Omega)=\left\{u\right.$ is a measurable real function on $\left.\Omega: \int_{\Omega}|u|^{p(x)} d x<\infty\right\}$.

We define a norm, the so-called Luxemburg norm, on

$$
L^{p(x)}(\Omega)
$$

by the formula

$$
|u|_{p(x)}=\inf \left\{\lambda>0: \int_{\Omega}\left|\frac{u(x)}{\lambda}\right|^{p(x)} d x \leq 1\right\}
$$

and then

$$
\left(L^{p(x)}(\Omega),|\cdot|_{p(x)}\right)
$$

becomes a Banach space.

Define the variable exponent Sobolev space by

$$
W^{1, p(x)}(\Omega)=\left\{u \in L^{p(x)}(\Omega) ;|\nabla u| \in L^{p(x)}(\Omega)\right\}
$$

then it can be equipped with the norm

$$
\|u\|_{1, p(x)}=|u|_{p(x)}+|\nabla u|_{p(x)}, \forall u \in W^{1, p(x)}(\Omega) .
$$

The space

$$
W_{0}^{1, p(x)}(\Omega)
$$

is defined as the closure of

$$
C_{0}^{\infty}(\Omega)
$$

In

$$
W^{1, p(x)}(\Omega)
$$

with respect to the norm

$$
\|u\|_{1, p(x)}
$$

For

$$
u \in W_{0}^{1, p(x)}(\Omega)
$$

we can define an equivalent norm

$$
\|u\|=|\nabla u|_{p(x)},
$$

since the well-known Poincaré inequality holds.

Proposition $1 .[8,14]$

The conjugate space of $L^{p(x)}(\Omega)$ is $L^{p^{\prime}(x)} \Omega$

where $\frac{1}{p(x)}+\frac{1}{p^{\prime}(x)}=1$.

For any $u \in L^{p(x)}(\Omega)$

and $v \in L^{p^{\prime}(x)}(\Omega)$ we have

$\left|\int_{\Omega} u v d x\right| \leq\left(\frac{1}{p^{-}}+\frac{1}{\left(p^{-}\right)^{\prime}}\right)|u|_{p(x)}|v|_{p^{\prime}(x)}$. 


$$
\begin{aligned}
& \text { P r oposition } 2 \cdot[8,14] \\
& \text { Denote } \rho(u)=\int_{\Omega}|u|^{p(x)} d x, \\
& \forall u, u_{n} \in L^{p(x)}(\Omega) \text {, then one has } \\
& (\text { i })|u|_{p(x)}>1 \Rightarrow|u|_{p(x)}{ }^{p^{-}} \leq \rho(u) \leq|u|_{p(x)}{ }^{p^{+}}, \\
& (\text {ii })|u|_{p(x)} \leq 1 \Rightarrow|u|_{p(x)} p^{p^{+}} \leq \rho(u) \leq|u|_{p(x)} p^{p^{-}}, \\
& (\text {iii }) \lim _{n \rightarrow \infty}\left|u_{n}\right|_{p(x)}=0 \Leftrightarrow \lim _{n \rightarrow \infty} \rho\left(u_{n}\right)=0, \\
& (\text { iii }) \lim _{n \rightarrow \infty}\left|u_{n}\right|_{p(x)} \rightarrow \infty \Leftrightarrow \lim _{n \rightarrow \infty} \rho\left(u_{n}\right) \rightarrow \infty . \\
& \quad \operatorname{Pr} \text { oposition } 3 .[8,14] \text { If } u, u_{n} \in L^{p(x)}(\Omega),
\end{aligned}
$$$$
\text { then the following statements are equivalent: }
$$$$
\text { (i) } \lim _{n \rightarrow \infty}\left|u_{n}-u\right|_{p(x)}=0 \text {, }
$$$$
\text { (ii) } \lim _{n \rightarrow \infty} \rho\left(u_{n}-u\right)=0 \text {, }
$$$$
\text { (iii) } u_{n} \rightarrow u \text { in measure } \Omega
$$$$
\text { and } \lim \rho\left(u_{n}\right)=\rho(u) \text {. }
$$

Proposition 4. [8,14](i) If $1<p^{-} \leq p^{+}<\infty$,

then the spaces $L^{p(x)}(\Omega), W^{1, p(x)}(\Omega)$

and $W_{0}^{1, p(x)}(\Omega)$ are separable and reflexive Banach spaces,

(ii) If $p_{1}, p_{2} \in C_{+}(\bar{\Omega})$ and $p_{1}(x) \leq p_{2}(x)$ for any $x \in \bar{\Omega}$, then the embedding $L^{p_{1}(x)}(\Omega) \rightarrow L^{p_{2}(x)}(\Omega)$ is continuous,

(iii) If $q \in C_{+}(\bar{\Omega})$ and $q(x)<p^{*}(x)$ for any $x \in \bar{\Omega}$, then

the embedding $W_{0}^{1, p(x)}(\Omega) \rightarrow L^{q(x)}(\Omega)$ is continuous and compact, where

$p^{*}(x):=\left\{\begin{array}{cc}\frac{N p(x)}{N-p(x)} & \text { if } p(x)<N, \\ +\infty & \text { f } p(x) \geq N .\end{array}\right.$

Proposition 5.[8,14] Let X be a B anach space and define the functional

$\Lambda=\int_{\Omega} \frac{|\nabla u|^{p(x)}}{p(x)} d x$. Then $\Lambda: X \rightarrow \mathbb{R}$ is convex.

The mapping $\Lambda^{\prime}: X \rightarrow X^{*}$

is a strictly monotone, bounded hom eomorphism, and of $\left(S_{+}\right)$type, nam ely

$u_{n} \rightarrow u$ (weakly) and $\varlimsup_{n \rightarrow \infty}\left\langle\Lambda^{\prime}\left(u_{n}\right), u_{n}-u\right\rangle \leq 0$

implies $u_{n} \rightarrow u($ strongly $)$.

Definition 6. Let $X$ be a Banach space and $I: X \rightarrow \mathbb{R}$ a $C^{1}$ - functional.

Wesay that a functional I satisfies the

Palais - Smale condition ( $(P S)$ for short)

if any sequence $\left\{u_{n}\right\}$ in $X$

such that $\left\{I\left(u_{n}\right)\right\}$ is bounded

and $I^{\prime}\left(u_{n}\right) \rightarrow 0$ as $n \rightarrow \infty$, admits a convergent subsequence.

We say that

$$
u \in W_{0}^{1, p(x)}(\Omega)
$$

is a weak solution of $(\mathrm{P})$ if

$$
m\left(\int_{\Omega} \frac{|\nabla u|^{p(x)}}{p(x)} d x\right) \int_{\Omega}|\nabla u|^{p(x)-2} \nabla u \nabla \varphi d x=\int_{\Omega} f(x, u) \varphi
$$

Where

$$
\varphi \in W_{0}^{1, p(x)}(\Omega)
$$

We associate to the problem (P) the energy functional, defined as

$$
\begin{gathered}
I: W_{0}^{1, p(x)}(\Omega) \rightarrow \mathbb{R}, \\
I(u)=M\left(\int_{\Omega} \frac{|\nabla u|^{p(x)}}{p(x)} d x\right)-\int_{\Omega} F(x, u),
\end{gathered}
$$

Where

$$
M(t)=\int_{0}^{t} m(s) d s
$$

and

$$
F(x, u)=\int_{0}^{u} f(x, s) d s
$$

We know that from (m1) and (f0) (see Section 3) $I$ is well defined and in a standard way we can prove that

$$
I \in C^{1}\left(W_{0}^{1, p(x)}(\Omega), \mathbb{R}\right)
$$

and that the critical points of I are solutions of $(\mathrm{P})$. Moreover, the derivative of $I$ is given by

$$
\begin{aligned}
& \left\langle I^{\prime}(u), \varphi\right\rangle=m\left(\int_{\Omega} \frac{|\nabla u|^{p(x)}}{p(x)} d x\right) \int_{\Omega}|\nabla u|^{p(x)-2} \nabla u \nabla \varphi d x \\
& -\int_{\Omega} f(x, u) \varphi
\end{aligned}
$$

for all

$$
u, \varphi \in W_{0}^{1, p(x)}(\Omega) .
$$

\section{Existence Results via Brouwer's Fixed Point Theorem}




$$
\begin{aligned}
& \text { Lemma } 7 \cdot[12] \text { Let } F: \mathbb{R}^{n} \rightarrow \mathbb{R}^{n} \text { be continuous } \\
& \text { such that }\langle F(x), x\rangle \geq 0 \text { for all } x \text { such } \\
& \text { that }|x|=R>0, \text { where }\langle\cdot, \cdot\rangle \text { is the usual inner } \\
& \text { product in } \mathbb{R}^{n} \text { and }|\cdot| \text { its related norm. } \\
& \text { Then there exists } x_{0} \text { such that } \\
& \left|x_{0}\right| \leq R \text { and } F\left(x_{0}\right)=0 .
\end{aligned}
$$

Now, we are ready to set and prove the first main result of the present paper.

Theorem 8 . Assume that the following

assumptions hold $:(m 0) m:(0,+\infty) \rightarrow(k,+\infty)$ is a continuous function with $k>0,(f 0) f: \Omega \times \mathbb{R} \rightarrow \mathbb{R}$ is Caratheodory function and satisfiesthe growth

condition $|f(x, t)| \leq c\left(1+|t|^{q(x)-1}\right) \forall(x, t) \in \Omega \times \mathbb{R}$, where $q \in C_{+}(\bar{\Omega})$ and $q(x)<p^{*}(x)$ for all $x \in \Omega$.

Further, assume that there exists a

positive real number a, $\alpha \in C_{+}(\bar{\Omega})$ with $\alpha^{+}<p^{-}$such

that $(f 1) f(x, t) t \leq a\left(1+|t|^{\alpha(x)}\right) \forall(x, t) \in \Omega \times \mathbb{R}$

such that

$$
a<\frac{k}{C^{\alpha^{+}}},
$$

where $C$ is embedding const of

$W_{0}^{1, p(x)}(\Omega) \rightarrow L^{\alpha(x)}(\Omega)$.Then problem $(P)$ has

at least one weak solution. Further,

any solution u of $(P)$ satisfies the estimate

$\|u\| \leq \frac{a|\Omega|}{k-a C^{\alpha^{+}}}$.

Proof. Because

$$
W_{0}^{1, p(x)}(\Omega)
$$

is a reflexive and separable Banach space, then there are

$$
\left\{w_{i}\right\} \subset W_{0}^{1, p(x)}(\Omega)
$$

And

$$
\left\{w_{i}^{*}\right\} \subset\left(W_{0}^{1, p(x)}(\Omega)\right)^{*}
$$

such that

$$
\begin{gathered}
W_{0}^{1, p(x)}(\Omega)=\overline{\operatorname{span}\left\{w_{i}: i=1,2, \ldots\right\}}, \\
\left(W_{0}^{1, p(x)}(\Omega)\right)^{*}=\overline{\operatorname{span}\left\{w_{i}^{*}: i=1,2, \ldots\right\}}
\end{gathered}
$$

And

$$
\left\langle w_{i}, w_{j}^{*}\right\rangle= \begin{cases}1, & i=j \\ 0, & i \neq j\end{cases}
$$

Define

$$
V_{n}=\operatorname{span}\left\{w_{1}, \ldots, w_{n}\right\}
$$

Then $V_{n}$ is isometric to $\mathbb{R}^{n}$. Let

$$
\xi=\left(\xi_{k}\right) \in \mathbb{R}^{n}
$$

To each such $\xi$, we associate a uniqe element $v \in V_{n}$ by the relation $v=\sum_{k=1}^{n} \xi_{k} w_{k}$. Further, since the $\left\{w_{k}\right\}$ are orthonormal in $W_{0}^{1, p(x)}(\Omega)$, we have

$$
\|v\|=\left(\sum_{k=1}^{n}\left|\xi_{k}\right|^{2}\right)^{1 / 2}=\mid \xi_{\mathbb{R}^{n}}
$$

We look for solutions $u_{n} \in V_{n}$ of the approximate problem

$$
\begin{aligned}
& m\left(\int_{\Omega} \frac{\left|\nabla u_{n}\right|^{p(x)}}{p(x)} d x\right) \int_{\Omega}\left|\nabla u_{n}\right|^{p(x)-2} \nabla u_{n} \nabla w_{k} d x \\
& =\int_{\Omega} f\left(x, u_{n}\right) w_{k} d x, k=1,2, \ldots
\end{aligned}
$$

To solve this algebraic system, we now define the operator

$$
P_{n}: \mathbb{R}^{n} \rightarrow \mathbb{R}^{n}
$$

by

$$
\begin{aligned}
& \left(P_{n} u\right)_{k}=m\left(\int_{\Omega} \frac{|\nabla u|^{p(x)}}{p(x)} d x\right) \int_{\Omega}|\nabla u|^{p(x)-2} \nabla u \nabla w_{k} d x \\
& -\int_{\Omega} f(x, u) w_{k} d x, \quad u \in V_{n} .
\end{aligned}
$$

By the condition ( $\mathrm{f} 0$ ), the growth of function $f$ is subcritical, so $u \rightarrow f(\cdot, u)$ defines a continuous Nemytskii mapping

$$
N_{f}: L^{\beta(x)}(\Omega) \rightarrow L^{\beta^{\prime}(x)}(\Omega)
$$

We note that $P_{n}$ is continuous from continuity of $m$ and $f(x, u)$ with respect to $u$. Therefore, from $(\mathrm{m} 0)$, (f0, (f1) and Poincaré inequality, for $u \in V_{n}$ with 


$$
\|u\|>1
$$

we get

$$
\begin{aligned}
& \left\langle P_{n} u, u\right\rangle \\
& \geq k\|u\|^{p^{-}}-\int_{\Omega} f(x, u) u d x \\
& \geq k\|u\|^{p^{-}}-a C^{\alpha^{+}}\|u\|^{\alpha^{+}}-a|\Omega| \\
& \geq\left(k-a C^{\alpha^{+}}\right)\|u\|^{p^{-}}-a|\Omega| .
\end{aligned}
$$

This shows, from (3.1), the existence of $R>0$ depending only on $k, a, C, \Omega$ such that $\left\langle P_{n} u, u\right\rangle \geq 0$ if $\|u\|=R$. Hence by consequence of Brouwer's fixed point theorem (Lemma 7), the system (3.2) has a solution $u_{n} \in V_{n}$ satisfying $\left\|u_{n}\right\| \leq R$. From this bound estimate, going to a subsequence if necessary, there exists a $u$ such that $u_{n} \rightarrow u($ weakly $)$ in $W_{0}^{1, p(x)}(\Omega)$.

Moreover, since $W_{0}^{1, p(x)}(\Omega) \rightarrow L^{\alpha(x)}(\Omega)$ is compactly and the mapping $N_{f}$ is continuous from

$$
L^{\alpha(x)}(\Omega) \rightarrow L^{\alpha^{\prime}(x)}(\Omega)
$$

(see e.g., [9]), we have

$$
\begin{aligned}
& u_{n} \rightarrow u\left(\text { strongly)in } L^{\alpha(x)}(\Omega),\right. \\
& f\left(x, u_{n}\right) \rightarrow f(x, u) \text { in } L^{\alpha^{\prime}(x)}(\Omega) .
\end{aligned}
$$

Then fixing $k$ in (3.2) and letting $n \rightarrow+\infty$, we conclude that

$$
\begin{aligned}
& m\left(\int_{\Omega} \frac{|\nabla u|^{p(x)}}{p(x)} d x\right) \int_{\Omega}|\nabla u|^{p(x)-2} \nabla u \nabla w_{k} d x \\
& =\int_{\Omega} f(x, u) w_{k} d x .
\end{aligned}
$$

From the completeness of $\left\{w_{k}\right\}$, the equality (3.3) holds with $w_{k}$ replaced by any $\varphi \in W_{0}^{1, p(x)}(\Omega)$, we get

$$
\begin{aligned}
& m\left(\int_{\Omega} \frac{|\nabla u|^{p(x)}}{p(x)} d x\right) \int_{\Omega}|\nabla u|^{p(x)-2} \nabla u \nabla \varphi d x \\
& =\int_{\Omega} f(x, u) \varphi d x,
\end{aligned}
$$

which means that $\mathrm{u}$ is a solution of $(\mathrm{P})$. Finally, if $u$ is any solution of $(\mathrm{P})$ and it is nontrivial, then

$$
m\left(\int_{\Omega} \frac{|\nabla u|^{p(x)}}{p(x)} d x\right) \int_{\Omega}|\nabla u|^{p(x)} d x=\int_{\Omega} f(x, u) d x .
$$

As a consequence, we have

$$
\|u\| \leq \frac{a|\Omega|}{k-a C^{\alpha^{+}}} .
$$

The proof is completed.

\section{Existence Results via Variational \\ Method}

In this section, we prove the existence of trivial and nontrivial weak solutions of problem (P) by using the Mountain-Pass lemma.

Theorem 9. Assumethat $\left(m_{0}\right)$ holds. Further,

if the growthcondition

$(f 2) f: \bar{\Omega} \times \mathbb{R} \rightarrow \mathbb{R}$ is Carathedory functionand satisfies

$$
|f(x, t)| \leq c\left(1+|t|^{\alpha-1}\right) \forall(x, t) \in \bar{\Omega} \times \mathbb{R},
$$

holds, where $1 \leq \alpha<p^{-}$, then problem $(P)$ has a weak solution.

Proof. From (f2) and (m0), we have $|F(x, t)| \leq c\left(|t|+|t|^{\alpha}\right)$ and $M(t) \geq k t$, respectively. Then, it follows

$$
\begin{aligned}
& I(u) \\
& =M\left(\int_{\Omega} \frac{|\nabla u|^{p(x)}}{p(x)} d x\right)-\int_{\Omega} F(x, u) \\
& \geq k \int_{\Omega} \frac{|\nabla u|^{p(x)}}{p(x)} d x-c \int_{\Omega}|u|^{\alpha} d x-c \int_{\Omega}|u| d x \\
& \geq \frac{k}{p^{+}}\|u\|^{p^{-}}-c\|u\|^{\alpha}-c\|u\| \rightarrow+\infty(\|u\| \rightarrow \infty) .
\end{aligned}
$$

This shows the coerciveness of $I$. Furthermore, since $I$ is weakly lower semicontinuous, it has a critical point $u$ in $W_{0}^{1, p(x)}(\Omega)$, which is a weak solution of $(\mathrm{P})$. The proof is completed. 
Theorem 10. Assume $\left(f_{0}\right)$ and the following assumptions hold :

$(m 1) m:(0,+\infty) \rightarrow(k,+\infty)$ is a continuous function with $k>0$, and

satisfies the growth condition $A t^{\gamma-1} \leq m(t) \leq B t^{\gamma-1}$, for all $t>0$, where $A, B$ and $\gamma$ are positive const such that $\frac{B}{A} \geq\left(p^{-}\right)^{\gamma-1}$ with $\gamma>1$;

$(f 3) f(x, t)=o\left(|t|^{\gamma p^{+}-1}\right),(t \rightarrow 0)$ uniformly for $x \in \Omega$, where $^{-}>\gamma p^{+}$;

(AR) Ambrosetti-Rabinowitz's condition holds, i.e.,

$\exists K>0, \theta>\frac{B}{A} \frac{\gamma\left(p^{+}\right)^{\gamma}}{\left(p^{-}\right)^{\gamma-1}}$ suchthat

$0<\theta F(x, t) \leq f(x, t) t,|t|>K$ a.e. $x \in \Omega$.

Then problem $(P)$ has a weak solution.

To obtain the results of Theorem 10, we need to verify the following two lemmas.

Lemma 11.Suppose (m1), (AR) and ( $f 0)$ hold.

Then I satisfies the (PS) condition.

Proof. Let assume that there exists a sequence $\left\{u_{n}\right\}$ in $W_{0}^{1, p(x)}(\Omega)$ such that

$$
\left|I\left(u_{n}\right)\right| \leq c \text { and } I^{\prime}\left(u_{n}\right) \rightarrow 0 .
$$

Therefore,

$$
\begin{aligned}
& c+\left\|u_{n}\right\| \\
\geq & I\left(u_{n}\right)-\frac{1}{\theta}\left\langle I^{\prime}\left(u_{n}\right), u_{n}\right\rangle \\
\geq & \frac{A}{\gamma\left(p^{+}\right)^{\gamma}}\left(\int_{\Omega}\left|\nabla u_{n}\right|^{p(x)} d x\right)^{\gamma}-\frac{B}{\theta\left(p^{-}\right)^{\gamma-1}}\left(\int_{\Omega}\left|\nabla u_{n}\right|^{p(x)} d x\right)^{\gamma-1} \\
\times & \left(\int_{\Omega}\left|\nabla u_{n}\right|^{p(x)} d x\right)+\int_{\Omega}\left(\frac{1}{\theta} f\left(x, u_{n}\right) u_{n}-F\left(x, u_{n}\right)\right) d x \\
\geq & \left(\frac{A}{\gamma\left(p^{+}\right)^{\gamma}}-\frac{B}{\theta\left(p^{-}\right)^{\gamma-1}}\right)\left(\int_{\Omega}\left|\nabla u_{n}\right|^{p(x)} d x\right)^{\gamma}-c \\
\geq & \left(\frac{A}{\gamma\left(p^{+}\right)^{\gamma}}-\frac{B}{\theta\left(p^{-}\right)^{\gamma-1}}\right)\left\|u_{n}\right\|^{\gamma p^{-}}-c .
\end{aligned}
$$

Therefore, $\left\{\left\|u_{n}\right\|\right\}$ is bounded in $W_{0}^{1, p(x)}(\Omega)$. From this bound estimate, going to a subsequence if necessary, there exists a $u$ in $W_{0}^{1, p(x)}(\Omega)$ such that

$$
u_{n} \rightarrow u(\text { weakly }) \operatorname{in} W_{0}^{1, p(x)}(\Omega)
$$

Thanks to the compact embedding

$$
W_{0}^{1, p(x)}(\Omega) \rightarrow L^{q(x)}(\Omega)
$$

we get

$$
\begin{aligned}
& u_{n} \rightarrow u(\text { strongly }) \text { in } L^{q(x)}(\Omega) \\
& u_{n} \rightarrow u \text { a.e. } x \in \Omega .
\end{aligned}
$$

By (4.1), we have

$$
\left\langle I^{\prime}\left(u_{n}\right), u_{n}\right\rangle \rightarrow 0
$$

Thus

$$
\begin{aligned}
& \left\langle I^{\prime}\left(u_{n}\right), u_{n}-u\right\rangle \\
& =m\left(\int_{\Omega} \frac{\left|\nabla u_{n}\right|^{p(x)}}{p(x)} d x\right) \int_{\Omega}\left|\nabla u_{n}\right|^{p(x)-2} \nabla u_{n}\left(\nabla u_{n}-\nabla u\right) d x \\
& -\int_{\Omega} f\left(x, u_{n}\right)\left(u_{n}-u\right) d x \rightarrow 0 .
\end{aligned}
$$

From (f0) and Proposition 1, it follows

$$
\begin{aligned}
& \left|\int_{\Omega} f\left(x, u_{n}\right)\left(u_{n}-u\right) d x\right| \leq \\
& \left.\left.c|| u_{n}\right|^{q(x)-1}\right|_{q^{\prime}(x)}\left|u_{n}-u\right|_{q(x)}+c \int_{\Omega}\left|u_{n}-u\right| d x .
\end{aligned}
$$

If we consider the relations given in (4.2), we get

$$
\int_{\Omega} f\left(x, u_{n}\right)\left(u_{n}-u\right) d x \rightarrow 0
$$

Hence

$$
m\left(\int_{\Omega} \frac{\left|\nabla u_{n}\right|^{p(x)}}{p(x)} d x\right) \int_{\Omega}\left|\nabla u_{n}\right|^{p(x)-2} \nabla u_{n}\left(\nabla u_{n}-\nabla u\right) d x \rightarrow 0 .
$$

From (m1), it follows

$$
\int_{\Omega}\left|\nabla u_{n}\right|^{p(x)-2} \nabla u_{n}\left(\nabla u_{n}-\nabla u\right) d x \rightarrow 0
$$

Since the functional (4.3) is of type $\left(S_{+}\right)$(Proposition 5), we get $u_{n} \rightarrow u($ strongly $)$ in $W_{0}^{1, p(x)}(\Omega) . I$ satisfies (PS) condition. 
Lemma 12.Suppose $(m 1),(A R),(f 0)$ and $(f 3)$ hold.

Then the following statements hold:

(i) There exist two psitive real numbers $\delta$ and $\mu$ such that

$I(u) \geq \mu>0, u \in W_{0}^{1, p(x)}(\Omega)$ with $\|u\|=\delta ;$

(ii) There exist a $\operatorname{in} W_{0}^{1, p(x)}(\Omega)$ such that $\|u\|>\delta, I(u)<0$.

\section{Proof}

(i) Let us assume

$$
\|u\|<1
$$

Then by $(\mathrm{m} 0)$, we have

$$
I(u)=\frac{A}{\gamma\left(p^{+}\right)^{\gamma}}\|u\|^{\lambda p^{+}}-\int_{\Omega} F(x, u) .
$$

By the continuous embeddings

$$
W_{0}^{1, p(x)}(\Omega) \rightarrow L^{q(x)}(\Omega)
$$

And

$$
W_{0}^{1, p(x)}(\Omega) \rightarrow L^{\gamma p^{+}}(\Omega)
$$

there exist some positive constants $c_{0}$ and $c_{1}$ such that,

$$
\forall u \in W_{0}^{1, p(x)}(\Omega),
$$

$$
|u|_{q(x)} \leq c_{0}\|u\| \text { and }|u|_{\gamma p^{+}} \leq c_{1}\|u\| \text {, }
$$

Let $\varepsilon>0$ be small enough such that

$$
\varepsilon c_{1}^{\gamma p^{+}}<\frac{A}{2 \gamma\left(p^{+}\right)^{\gamma}} .
$$

Then, using (f0) and (f3), we get

$$
F(x, t) \leq \varepsilon|t|^{\gamma p^{+}}+c_{\varepsilon}|t|^{q(x)}, \forall(x, t) \in \Omega \times \mathbb{R} .
$$

Therefore, by (m1) and (4.4), it follows

$$
\begin{aligned}
& I(u) \\
& \geq \frac{A}{\gamma\left(p^{+}\right)^{\gamma}}\|u\|^{\gamma p^{+}}-\varepsilon \int_{\Omega}|u|^{\gamma p^{+}} d x-c_{\varepsilon} \int_{\Omega}|u|^{q(x)} d x \\
& \geq \frac{A}{\gamma\left(p^{+}\right)^{\gamma}}\|u\|^{\gamma p^{+}}-\varepsilon c_{1}^{\gamma p^{+}}\|u\|^{\gamma p^{+}}-c_{\varepsilon} c^{q^{-}}{ }_{0}\|u\|^{q^{-}} \\
& \geq\left(\frac{A}{\gamma\left(p^{+}\right)^{\gamma}}-\varepsilon c_{1}^{\gamma p^{+}}\right)\|u\|^{\gamma p^{+}}-c\|u\|^{q^{-}} .
\end{aligned}
$$

Since $\|u\|<1$ and $q^{-}>\gamma p^{+}$, there exist positive real numbers $\delta$ and $\mu$ such that

$$
I(u) \geq \mu>0, u \in W_{0}^{1, p(x)}(\Omega)
$$

With

$$
\|u\|=\delta \in(0,1)
$$

(ii) From (AR), one easily deduces

$$
F(x, t) \geq c|t|^{\theta},|t| \geq K \text { a.e. } x \in \Omega .
$$

Moreover, when $t>1$ is large enough, from (m1), we obtain that

$$
M(t) \leq \frac{B}{\gamma} t^{\gamma} \leq \frac{B}{\gamma} t^{\gamma p^{+}} .
$$

Hence, for $\omega \in W_{0}^{1, p(x)}(\Omega), \omega \neq 0$, and $t>1$ we have

$$
\begin{aligned}
& I(t \omega)=M\left(\int_{\Omega} \frac{\mid \nabla t \omega^{p(x)}}{p(x)} d x\right)-\int_{\Omega} F(x, t \omega) \\
& \leq \frac{B}{\gamma\left(p^{-}\right)^{\gamma}} t^{\gamma p^{+}}\|\omega\|^{\gamma p^{+}}-c t^{\theta} \int_{\Omega}|\omega|^{\theta} d x-c .
\end{aligned}
$$

From (m1) and (AR), it can be obtained that $\theta>\gamma p^{+}$. Therefore

$$
I(t \omega) \rightarrow-\infty(t \rightarrow+\infty) .
$$

Proof of Theorem 10. From Lemma 11, Lemma 12 and the fact that $I(0)=0, I$ satisfies the Mountain Pass theorem (see e.g.,[20]). Therefore, $I$ has at least one nontrivial critical point, i.e., (P) has a nontrivial weak solution. We are done.

\section{References}

[1] S. N. Antontsev, J. F. Rodrigues, "On stationary thermo-rheological viscous flows", Ann. Univ. Ferrara, Sez. 7, Sci. Mat., vol.52, pp. 19-36, 2006.

[2] M. Avci, B. Cekic, R. A. Mashiyev, "Existence and multiplicity of the solutions of the $\mathrm{p}(\mathrm{x})$-Kirchhoff type equation via genus theory", Math. Methods in the Applied Sciences, vol. 34 (14), pp.1751-1759, 2011.

[3] B. Cekic, A. V. Kalinin, R. A. Mashiyev, M. Avci, " $\mathrm{L}^{\wedge}\{\mathrm{p}(\mathrm{x})\}(\Omega)$-estimates of vector fields and some applications to magnetostatics problems", Journal of Math. Analysis and Appl., vol. 389 (2), pp. 838-851, 2012.

[4] F. J. S. A. Corrêa, G. M. Figueiredo, "On a elliptic equation of p-kirchhoff type via variational methods", Bull. Austral. Math. Soc., vol. 74, pp.263-277, 2006.

[5] G. Dai, D. Liu, "Infinitely many positive solutions for a p(x)-Kirchhoff-type equation”, J. Math. Anal. Appl., vol.359 (2), pp.704-710, 2009. 
[6] G. Dai, R. Hao, "Existence of solutions for a p(x)-Kirchhoff-type equation", J. Math. Anal. Appl., vol. 359 (2), pp. 275-284, 2009.

[7] X. L. Fan., "On nonlocal p(x)-Laplacian Dirichlet problems", Nonlinear Anal. vol.72, pp. 3314-3323, 2010.

[8] X. L. Fan, D. Zhao, "On the spaces $\mathrm{L}^{\wedge}\{\mathrm{p}(\mathrm{x})\}$ and $\mathrm{W}^{\wedge}\{\mathrm{m}, \mathrm{p}(\mathrm{x})\} ”$, J. Math. Anal. Appl., vol. 263, pp. 424-446, 2001 .

[9] X. Fan, Q. H. Zhang, "Existence of solutions for p(x)-Laplacian Dirichlet problems", Nonlinear Anal., vol. 52, pp. 1843-1852, 2003.

[10] P. A. Hästö, "On the variable exponent Dirichlet energy integral", Comm. Pure Appl. Anal., vol. 5 (3), pp. 413-420, 2006.

[11] X. He, W. Zou, "Infinitely many positive solutions for Kirchhoff-type problems", Nonlinear Anal. vol. 70, pp. 1407-1414, 2009.

[12] S. Kesavan, Topics in Functional Analysis and Applications, Wiley, NewYork, 1989.

[13] G. Kirchhoff, Mechanik, Teubner, Leipzig, 1883.

[14] O. Kovăčik, J. Răkosnik, "On spaces $\mathrm{L}^{\wedge}\{\mathrm{p}(\mathrm{x})\}$ and $\mathrm{W}^{\wedge}\{1, \mathrm{p}(\mathrm{x})\} ”$, Czechoslovak Math. J. vol. 41 (116), pp. 592-618, 1991 .
[15] T. F. Ma, "Remarks on an elliptic equation of Kirchhoff type", Nonlinear Anal., vol. 63, pp. 1967-1977, 2005.

[16] H. Ma, G. Dai, "Existence results for a variable exponent elliptic problem via topological method", Boundary Value Problems, vol. 2012:99. doi:10.1186/1687-2770-2012-99.

[17] M. Mihăilescu, V. Rădulescu, "On a nonhomogeneous quasilinear eigenvalue problem in Sobolev spaces with variable exponent", Proceedings Amer. Math. Soc., vol. 135 (9), pp. 2929-2937, 2007.

[18] S. Ogras, R. A. Mashiyev, M. Avci, Z. Yucedag, "Existence of Solutions for a Class of Elliptic Systems in $\mathbb{R}^{\mathrm{n}}$ Involning $(\mathrm{p}(\mathrm{x}), \mathrm{q}(\mathrm{x}))$-Laplacian", Journal of Inequalities and Applications, ID612938, pp.20, 2008.

[19] M. Růžička, Electrorheological fluids: Modeling and Mathematical Theory, Lecture Notes in Mathematics, Springer-Verlag: Berlin, 2000.

[20] M. Willem, Minimax Theorems, Birkhäuser, Boston, 1996.

[21] F. Wang, Y. An, "Existence of Nontrivial Solution for a Nonlocal Elliptic Equation with Nonlinear Boundary Condition", Boundary Value Problems Vol. 2009, Article ID 540360, 8 pages, doi:10.1155/2009/540360.

[22] V.V. Zhikov, "Averaging of functionals of the calculus of variations and elasticity theory", Math. USSR. Izv., vol. 9, pp. 33-66, 1987. 